\title{
Research Article \\ Effect of a High Magnetic Field on Eutectoid Point Shift and Texture Evolution in 0.81C-Fe Steel
}

\author{
Y. D. Zhang, ${ }^{1,2}$ C. Esling, ${ }^{2}$ M. Calcagnotto, ${ }^{2,3}$ M. L. Gong, ${ }^{1}$ H. Klein, ${ }^{3}$ X. Zhao, ${ }^{1}$ and L. Zuo ${ }^{1}$ \\ ${ }^{1}$ Key Laboratory for Anisotropy and Texture of Materials, Northeastern University, Shenyang 110004, China \\ ${ }^{2}$ Laboratoire d'Etude des Textures et Application aux Matériaux (LETAM), CNRS UMR 7078, University of Metz, \\ Ile du Saulcy, Metz 57045, France \\ ${ }^{3}$ Department of Crystallography, University of Goettingen, 37077 Goettingen, Germany
}

Correspondence should be addressed to C. Esling, claude.esling@univ-metz.fr

Received 3 July 2007; Accepted 16 January 2008

Recommended by Richard Penelle

A $12 \mathrm{~T}$ magnetic field has been applied to the annealing process of a $0.81 \% \mathrm{C}-\mathrm{Fe}$ (wt.\%). It is found that the magnetic field shifts the eutectoid carbon content from $0.77 \mathrm{wt} . \%$ to $0.83 \mathrm{wt} . \%$. The statistical thermodynamic calculations were performed to calculate the eutectoid temperature change by the magnetic field. Calculation shows that the increase of the eutectoid temperature by a $12 \mathrm{~T}$ field is $29^{\circ} \mathrm{C}$. Synchrotron radiation measurements were performed to measure the pole figures of the samples and were analyzed by MAUD to determine the bulk texture of the ferrite phase in the field-treated and non-field-treated samples. Results show that although there is no specific preferred orientation appearing by applying the magnetic field, slight enhancement of $\langle 001\rangle$ fiber component occurs in both the sample normal direction (ND) and the transverse direction (TD). This effect might be related to the magnetic dipolar interaction between Fe atoms in the transverse field direction.

Copyright (C) 2008 Y. D. Zhang et al. This is an open access article distributed under the Creative Commons Attribution License, which permits unrestricted use, distribution, and reproduction in any medium, provided the original work is properly cited.

\section{INTRODUCTION}

Study of the effect of a high magnetic field on diffusional phase transformation in steels has attracted attention in recent years. So far most efforts have been made on revealing the effect of a magnetic field on phase transformation behaviors and microstructural characters in hypoeutectoid steels [1-8]. Results have shown that the magnetic field applied can increase the austenite-to-ferrite transformation temperature [1-3], accelerate the transformation process [5-7], and change the morphology and grain boundary character distribution of the product phases $[5,8]$. In this work, a quasi-eutectoid steel, 0.81C-Fe, was selected and heat-treated without and with a $12 \mathrm{~T}$ magnetic field. The shift of the eutectoid point by the application of a high magnetic field was experimentally investigated and theoretically calculated. The influence of the magnetic field applied on the evolution of the texture of ferrite has been investigated by means of synchrotron radiation measurements.

\section{EXPERIMENTAL}

The material used in this study was a plain eutectoid steel with chemical composition (wt.\%) $0.81 \% \mathrm{C}, 0.22 \% \mathrm{Si}$, $0.20 \% \mathrm{Mn}, 0.05 \% \mathrm{Cr}, 0.014 \mathrm{Cu}, 0.019 \% \mathrm{P}, 0.003 \% \mathrm{~S}$, and bal. Fe. It was prepared by induction casting. The cast rod was twice forged in the longitudinal direction followed by the radial direction, in the temperature range $1100-868^{\circ} \mathrm{C}$ to homogenize and refine the microstructure. Samples of dimensions $30 \mathrm{~mm} \times 10 \mathrm{~mm} \times 2 \mathrm{~mm}$ were cut from the hot forged bar. They were austenitized at $830^{\circ} \mathrm{C}$ for 50 minutes and cooled at a rate of $2,5,10$, and $23^{\circ} \mathrm{C} / \mathrm{min}$ without and with a 12-Tesla high magnetic field. During the heat treatment, the samples were placed in the central (zero magnetic force) region (200 $\mathrm{mm}$ long in the field direction).

The transformed microstructure is observed with an Olympus BX61 microscope equipped with a digital camera and the analysis software package for materials science. The crystal structures of proeutectoid ferrite and proeutectoid cementite are confirmed by the indexing of the EBSD Kikuchi 
patterns acquired with a Jeol JSM 6500F FEG-SEM equipped with the HKL Channel 5 EBSD system. The area percentages of bulk ferrite appearing in the magnetic field-treated samples and the lamellar spacing of pearlite in both the fieldand the non-field-treated samples were measured. The measurement was performed in 20 images of each sample to obtain a statistical representation of the results. As the lamellar spacing in each micrograph varies due to both the changing of formation temperature and the angles at which the lamellae intersect the sample-sectioning plane, the smallest lamellar spacing in each micrograph was measured. This smallest lamellar spacing obtained is related either to the pearlite formed at the very end of the transformation or to the angle, close to right angle, at which lamellae intersect the sample plane.

Synchrotron radiation measurements were performed at the Hamburg Synchrotron Radiation Laboratory (HASYLAB), German Electron Synchrotron (DESY), Hamburg, Germany, to measure the incomplete pole figures of the samples cooled at $2^{\circ} \mathrm{C} / \mathrm{min}$ without and with a 12 -Tesla high magnetic field. The measurements were carried out in transmission mode with a monochromatic synchrotron beam [9] of wavelength $0.164 \AA$ ( $75.59 \mathrm{keV})$. The collimator was set to an area of $1 \times 1 \mathrm{~mm}$. The first slit was set to $1 \times 1 \mathrm{~mm}$ and the second was set to $2 \times 2 \mathrm{~mm}$. During the measurement, the sample was rotated from $-80^{\circ}$ to $+80^{\circ}$ around the $\omega$ axis with a step size of $2^{\circ} /$ step and a measurement time of 4 s/step. Data were recorded with an area detector $34.5 \mathrm{~cm}$ in diameter) placed behind the sample, at a distance of $115.5 \mathrm{~cm}$. The data were analyzed with MAUD ("Materials Analysis Using Diffraction" program package by Lutterotti et al., University of Trento $[10,11])$ and represented in inverse pole figures.

\section{RESULTS AND DISCUSSION}

\subsection{The eutectoid point shift under a $12 \mathrm{~T}$ magnetic field}

Figure 1 shows the microstructures of samples cooled at $2^{\circ} \mathrm{C} / \mathrm{min}$ without (a) and with (b) a $12 \mathrm{~T}$ magnetic field. Besides the main constituent of lamellar pearlite, the striking difference between the two micrographs is that in the non-field-treated specimen we observed a slight amount of proeutectoid cementite (arrowed in the zoom image in the top right corner of Figure 1(a)), which characterizes the hypereutectoid microstructure, while in the field-treated specimen we find some bulk ferrite between pearlite colonies (white areas circled in Figure 1(b)) that is the typical component of hypoeutectoid microstructure, instead of proeutectoid cementite. The crystal structures of proeutectoid cementite and the proeutectoid ferrite observed with the optical microscope were further confirmed by the indexing of their Kikuchi patterns by EBSD technique. The average area percentages of the bulk ferrite measured in the field-treated specimens at various cooling rates are shown in Table 1. It is seen that the amount of the bulk ferrite decreases with the increase of the cooling rate. This is the normal influence of the cooling rate on proeutectoid transformation. The presence of the bulk ferrite in the field-treated specimens sug-

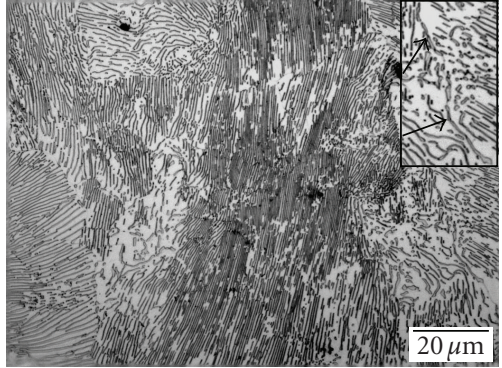

(a)

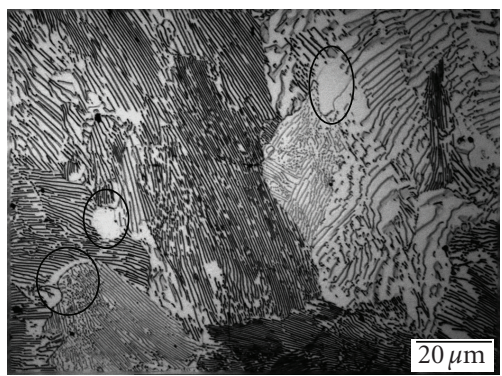

(b)

FIgURE 1: Optical micrographs of specimens austenitized at $830^{\circ} \mathrm{C}$ for 50 minutes and cooled at $2^{\circ} \mathrm{C} / \mathrm{min}$ without (a) and with (b) a $12 \mathrm{~T}$ magnetic field (the field direction is horizontal). The zoom image in the right-hand corner of (a) shows the secondary cementite, as indicated by the arrow. The magnification is 1.5 times that of the main image. The circles in (b) mark out the proeutectoid ferrite between pearlite colonies.

gests that the magnetic field shifts the eutectoid point of the Fe-C binary system beyond the carbon content of the tested material $(0.81 \% w t . C)$. Using the lever law, the carbon contents of the new eutectoid point under various cooling rates were calculated and displayed also in Table 1. As the carbon content of the equilibrium eutectoid point is obtained under equilibrium transformation condition, that is, under extreme slow cooling condition, the variation of the eutectoid carbon content with cooling rate is plotted in Figure 2 and extrapolated to zero cooling rate to obtain the equilibrium eutectoid carbon content. The extrapolated equilibrium eutectoid carbon content is $0.8287 \%$ (wt.). This value is noticeably different from that calculated in [3] (0.795 wt.\%).

The lamellar spacing of samples fully austenitized and cooled at various cooling rates $\left(2,5,10\right.$, and $\left.23^{\circ} \mathrm{C} / \mathrm{min}\right)$ without and with a $12 \mathrm{~T}$ magnetic field was also measured and is shown in Figure 3. It is seen that, at all the cooling rates tested, the lamellar spacing obtained is larger with the magnetic field than without. As the austenite to pearlite transformation is diffusional and involves the formation of the carbon-depleted ferrite and carbon-rich cementite, the lamellar spacing of pearlite depends on the diffusion length of carbon atoms that is temperature related. Therefore, the larger spacing corresponds to the higher formation temperature. This lamellar spacing increase resulting from the magnetic field applied evidences that the magnetic field also shifts 
TABLE 1: Average area percentages of the bulk ferrite and the standard deviation in $0.81 \mathrm{C}-\mathrm{Fe}$ specimens austenitized at $830^{\circ} \mathrm{C}$ for 50 minutes and cooled at various cooling rates with a $12 \mathrm{~T}$ magnetic field, and the calculated eutectoid carbon content by using lever law and the standard deviation.

\begin{tabular}{lll}
\hline $\begin{array}{l}\text { Cooling rate } \\
\left({ }^{\circ} \mathrm{C} / \mathrm{min}\right)\end{array}$ & $\begin{array}{l}\text { Area percent- } \\
\text { age of ferrite } \\
(\%)\end{array}$ & $\begin{array}{l}\text { Eutectoid } \\
\text { carbon content } \\
(\mathrm{wt} . \%)\end{array}$ \\
\hline 2 & $2.153 \pm 0.850$ & $0.827 \pm 0.0070$ \\
5 & $1.905 \pm 0.857$ & $0.825 \pm 0.0071$ \\
10 & $1.519 \pm 0.372$ & $0.822 \pm 0.0030$ \\
23 & $1.277 \pm 0.452$ & $0.820 \pm 0.0037$ \\
\hline
\end{tabular}

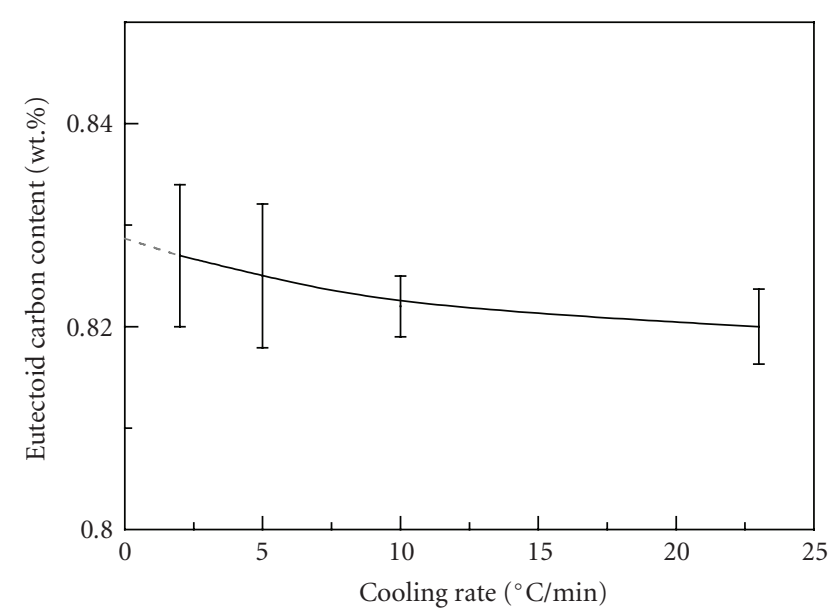

FIGURE 2: Eutectoid carbon content change with cooling rate. Black solid line: from the measured result. Gray line: extrapolation to the slow cooling rates following the changing tendency.

the eutectoid point to the high temperature side. As it is almost impossible to measure-in temperature scale-the eutectoid point shift in situ under a high magnetic field, its determination through theoretical calculation may be useful.

Let us remind that the eutectoid point of $\mathrm{Fe}-\mathrm{C}$ system is the intersection of the $\mathrm{Ae}_{3}$ line (austenite/ferrite $\gamma / \alpha$ equilibrium boundary) and the $\mathrm{Ae}_{\mathrm{cm}}$ line (austenite/cementite $\gamma /$ cem equilibrium line). By using the well-established statistical thermodynamic models, the $\mathrm{Ae}_{3}$ and $\mathrm{Ae}_{\mathrm{cm}}$ lines can be calculated, so the eutectoid composition and temperature can be determined. It is known that the driving force for austenite to proeutectoid ferrite transformation, $\Delta G^{\gamma \rightarrow \alpha+\gamma}$, is directly related to the equilibrium position (composition and temperature) of $\mathrm{Ae}_{3}$ line and is expressed as follows:

$$
\Delta G^{\gamma \rightarrow \alpha+\gamma}=R T\left[x^{\gamma} \ln \frac{a_{\mathrm{C}}^{\gamma / \alpha}}{a_{\mathrm{C}}^{\gamma}}+\left(1-x^{\gamma}\right) \ln \frac{a_{\mathrm{Fe}}^{\gamma / \alpha}}{a_{\mathrm{Fe}}^{y}}\right],
$$

where $x^{y}$ is the mole fraction of carbon in the initial austenite, $R$ is the gas constant, $T$ is absolute temperature, $a_{j}^{\gamma / \alpha}$ is the activity of $\mathrm{C}$ or Fe in austenite on the $\gamma / \alpha$ boundary, and $a_{j}^{\gamma}$ is the activity of $\mathrm{C}$ or $\mathrm{Fe}$ in the initial austenite. By definition, the driving force of a phase transformation is the

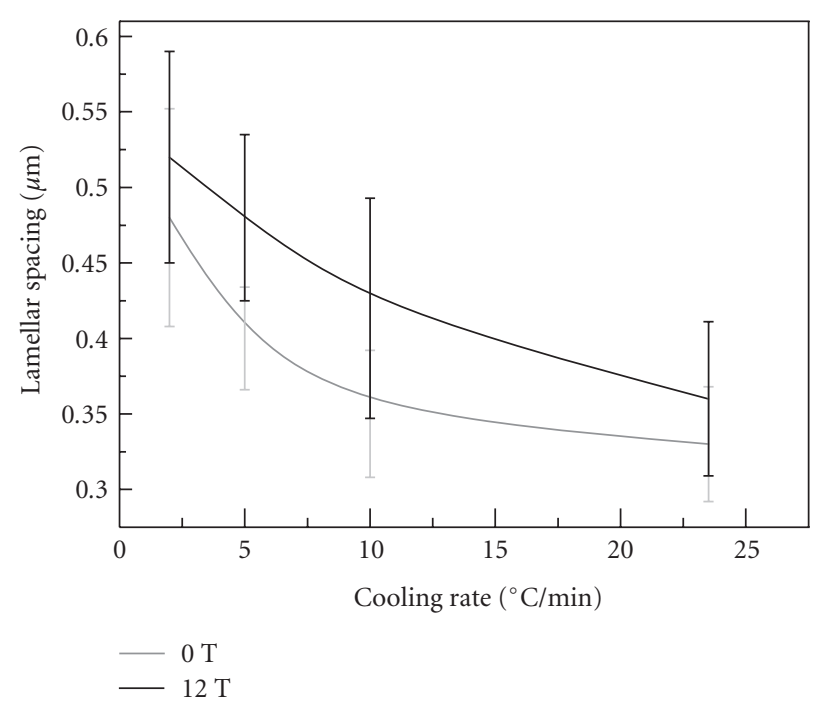

FIGURE 3: Average lamellar spacing of specimens austenitized at $830^{\circ} \mathrm{C}$ for 50 minutes and cooled at a rate of $2,5,10$, and $23^{\circ} \mathrm{C} / \mathrm{min}$ without and with a $12 \mathrm{~T}$ magnetic field.

Gibbs free energy difference between the product and parent phase. It is known that a magnetic field lowers the Gibbs free energy of a magnetized phase [5-7] according to its magnetization. The amount lowered is $\int_{0}^{M} \vec{B} \cdot d \vec{M}$ for ferromagnetic phase [12] and $\left(1 / 2 \mu_{0}\right) \chi B^{2}$ for paramagnetic phase [12], where $B=B_{0}+\mu_{0} M, B_{0}$ is the magnetic induction of the applied field, $\mu_{0}$ is the permeability of free space, $M$ is the magnetization, and $\chi$ is the magnetic susceptibility. As ferrite (or cementite) and austenite have different magnetization under the same magnetic field, their Gibbs free energy changes by the magnetic field are different. In this way, the phase equilibrium between the parent and the product phase is altered. Assume that under the magnetic field, the $\gamma / \alpha$ (or $\gamma / \mathrm{cem}$ ) boundary $x^{\gamma / i}$ (mole fraction of carbon in austenite on $\mathrm{Ae}_{3}$ or $\mathrm{Ae}_{\mathrm{cm}}$ line) moves to ${ }^{M} x^{\gamma / i}$. The activity of $\mathrm{C}$ or Fe in austenite at the new $\gamma / \alpha$ (or $\gamma / \mathrm{cem})$ boundary is ${ }^{M} a_{j}^{\gamma / i}$, thus the total driving force under the magnetic field for the austenite-to-proeutectoid ferrite transformation can be expressed as

$$
\Delta G^{\gamma \rightarrow \alpha+\gamma}+{ }^{M} \Delta G^{\gamma \rightarrow \alpha+\gamma}=R T\left[x^{\gamma} \ln \frac{{ }^{M} a_{\mathrm{C}}^{\gamma / \alpha}}{a_{\mathrm{C}}^{\gamma}}+\left(1-x^{\gamma}\right) \ln \frac{{ }^{M} a_{\mathrm{Fe}}^{\gamma / \alpha}}{a_{\mathrm{Fe}}^{\gamma}}\right] .
$$

Substituting (1) into (2) and letting $x^{y}$ take the equilibrium carbon content at the $\gamma / \alpha$ boundary $x^{\gamma / \alpha}$, we obtain

$$
{ }^{M} \Delta G^{\gamma \rightarrow \alpha+\gamma}=R T\left[x^{\gamma / \alpha} \ln \frac{{ }^{M} a_{\mathrm{C}}^{\gamma / \alpha}}{a_{\mathrm{C}}^{\gamma / \alpha}}+\left(1-x^{\gamma / \alpha}\right) \ln \frac{{ }^{M} a_{\mathrm{Fe}}^{\gamma / \alpha}}{a_{\mathrm{Fe}}^{\gamma / \alpha}}\right] .
$$

As for $\gamma /$ cem boundary ${ }^{M} x^{\gamma / \mathrm{Fe}_{3} \mathrm{C}}$, it can be obtained according to the way to obtain $x^{\gamma / \mathrm{Fe}_{3} \mathrm{C}}$ in [13] as follows:

$$
\Delta G^{\mathrm{Fe}_{3} \mathrm{C}}+{ }^{M} \Delta G^{\mathrm{Fe}_{3} \mathrm{C}-\gamma}=R T \ln ^{M} a_{\mathrm{C}}^{\gamma / \mathrm{Fe}_{3} \mathrm{C}}+3 R T \ln ^{M} a_{\mathrm{Fe}}^{\gamma / \mathrm{Fe}_{3} \mathrm{C}},
$$


where $\Delta G^{\mathrm{Fe}_{3} \mathrm{C}}=39828-193.296 T+22.345 T \ln T[14]$, the Gibbs free energy change of the formation of $1 \mathrm{~mol}$ cementite from $3 \mathrm{~mol}$ of $\gamma$-Fe and $1 \mathrm{~mol}$ of graphite, and ${ }^{M} \Delta G^{\mathrm{Fe}_{3} \mathrm{C}-\gamma}$ is magnetic Gibbs free energy difference between cementite and austenite that is determined by the magnetic susceptibility difference between the two phases. From the quasi-chemical model [15-17], the general expression of the activity of carbon and iron in austenite is

$$
\begin{aligned}
& \ln a_{\mathrm{C}}^{\gamma}=5 \ln \frac{1-2 x^{\gamma}}{x^{\gamma}}+\frac{6 w_{\gamma}}{R T}+6 \ln \left(\frac{\delta_{\gamma}-1+3 x^{\gamma}}{\delta_{\gamma}+1-3 x^{\gamma}}\right)+\frac{\Delta \bar{H}_{\gamma}-\Delta \bar{S}_{\gamma}^{x s} T}{R T}, \\
& \ln a_{\mathrm{Fe}}^{\gamma}=5 \ln \frac{1-x^{\gamma}}{1-2 x^{\gamma}}+6 \ln \left[\frac{1-2 J_{\gamma}+\left(4 J_{\gamma}-1\right) x^{\gamma}-\delta_{\gamma}}{2 J_{\gamma}\left(2 x^{\gamma}-1\right)}\right],
\end{aligned}
$$

where $\delta_{\gamma}=\left[1-2\left(1+2 J_{\gamma}\right) x^{\gamma}+\left(1+8 J_{\gamma}\right)\left(x^{\gamma}\right)^{2}\right]^{1 / 2}$ and $J_{\gamma}=$ $1-\exp \left(-w_{\gamma} / R T\right), w_{\gamma}$ is the pairwise interaction energy of carbon in austenite, $\Delta \bar{H}_{\gamma}$ and $\Delta \bar{S}_{\gamma}^{x s}$ are the relative partial enthalpy and nonconfigurational entropy of austenite. We finally take $w_{\gamma}=5880 \mathrm{~J} / \mathrm{mol}, \Delta \bar{H}_{\gamma}=44400$, and $\Delta \bar{S}_{\gamma}^{x s}=17.2$ from Shiflet et al. [18] (after trying various sets of data from published literature and verifying with the $\mathrm{Fe}-\mathrm{C}$ diagram without a magnetic field). By using a model based on the itinerant electron theory as implemented by Sakoh and Shimizu [19], a density functional-based method in conjunction with statistical approximations [20] and the Weiss molecular field model for ferromagnetism [21], the temperature variations of susceptibilities of austenite and cementite and the magnetization of ferrite can be calculated, and thus ${ }^{M} \Delta G^{\gamma \rightarrow \alpha+\gamma}$ and ${ }^{M} \Delta G^{\mathrm{cem}-\gamma}$ can be obtained. Substituting (5) into (3) and (4) and using the Newton-Raphson iterative algorithm, part of the phase diagram of $\mathrm{Fe}-\mathrm{C}$ binary system without and with a $12 \mathrm{~T}$ magnetic field was calculated and is shown in Figure 4. For comparison and completion, the $\gamma / \alpha$ and $\gamma /$ cem equilibrium lines without magnetic field are also calculated by using related equations [13] and the $\alpha / \gamma$ equilibrium lines without and with magnetic field are also calculated by using the related equations [13] and data [22] and are shown in the same figure. It is seen that the influence of the magnetic field is mainly on $\mathrm{Ae}_{3}$ line. The calculated eutectoid carbon content and temperature without and with a $12 \mathrm{~T}$ magnetic field is $0.779 \mathrm{wt} . \% \mathrm{C} ; 725.71^{\circ} \mathrm{C}$ and $0.847 \mathrm{wt} . \% \mathrm{C} ; 754.68^{\circ} \mathrm{C}$. The calculated eutectoid carbon contents without and with the magnetic field are quite close to the values determined from the experimental results. This agreement shows that the eutectoid temperature increase calculated by this method is reliable. In the case of a $12 \mathrm{~T}$ magnetic field, the eutectoid temperature shift is $28.97^{\circ} \mathrm{C}$. Equations (3) and (4) together with (5) offer a practical and accurate method to determine the new phase equilibrium boundary lines and the eutectoid shift under the influence of a magnetic field.

\subsection{Texture evolution under the effect of the applied magnetic field}

Figure 5 shows the inverse pole figures of the samples heattreated at slow cooling rate $\left(2^{\circ} \mathrm{C} / \mathrm{min}\right)$ without and with the

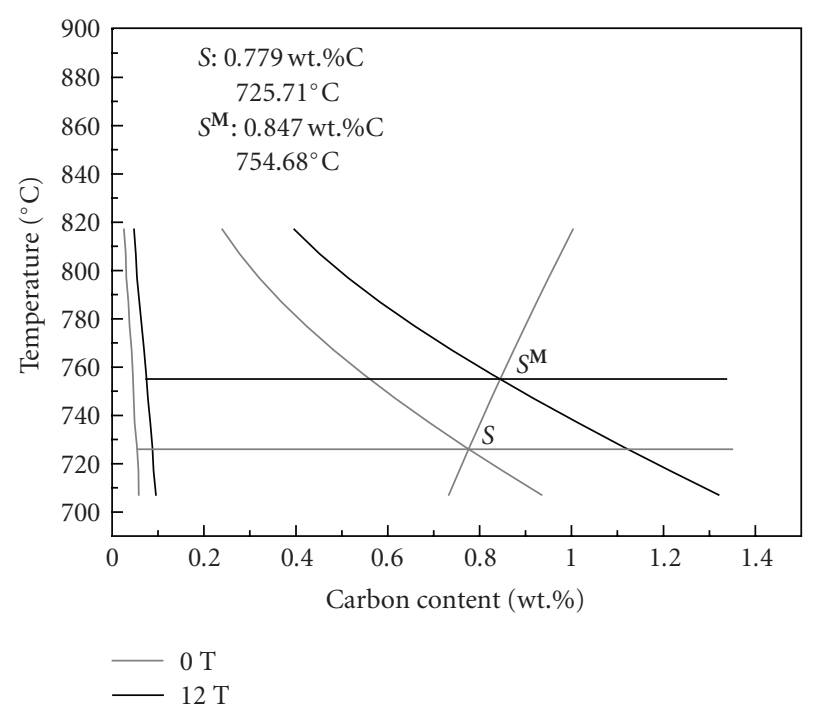

FIgUre 4: The calculated Fe-C phase diagram without and with a $12 \mathrm{~T}$ magnetic field.

12 T magnetic field and the corresponding sample coordinate system. Despite the textures are relatively weak, it is clearly seen that under the magnetic field there is slight enhancement of $\langle 001\rangle$ fiber component in both the sample normal direction (ND) and the widthwise or transverse direction (TD), as seen in Figure 5(b). This result corroborates earlier results we found in a medium carbon plain steel heat-treated under a $12 \mathrm{~T}$ magnetic field [8]. Actually in the present case, both ND and TD are transverse field directions. It is known that each Fe atom carries a magnetic moment. Under the applied magnetic field, these moments tend to align along the field direction. Then, there exists the dipolar interaction between neighboring Fe atoms. They attract each other along the field direction but repel each other along the transverse field directions (ND and TD in the present study). Correlatively, the distance between neighboring atoms tends to decrease along FD and increase along ND and TD to minimize the total energy of the system. For ferrite, the carbon atoms are located in the octahedral interstices, as shown in Figure 6 [8]. The interstices are flat in the $\langle 001\rangle$ direction. The occupation of the carbon atom in this interstice exerts an expansion stress on its neighboring iron atoms along the $\langle 001\rangle$ direction. This gives rise to the lattice distortion and creates distortion energy. If such a $\langle 001\rangle$ direction of a grain was parallel to the transverse field direction (ND or TD), the lattice distortion energy would be reduced through increasing the atomic spacing in such $\langle 001\rangle$ direction by the magnetic field. Therefore, the nucleation and growth of the grains having such $\langle 001\rangle$ parallel to the ND and TD are energetically favored by the magnetic field. In this way, the $\langle 001\rangle$ component is enhanced in the transverse field directions (ND and TD).

\section{SUMMARY}

The eutectoid carbon content and temperature of $\mathrm{Fe}-\mathrm{C}$ binary system under a magnetic field have been examined 


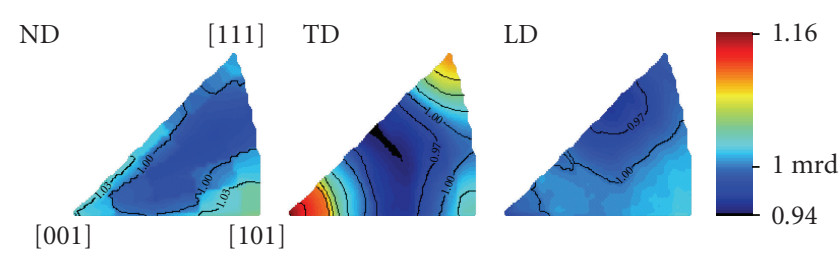

(a)
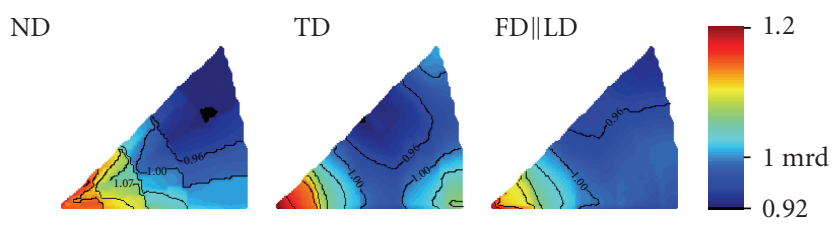

(b)

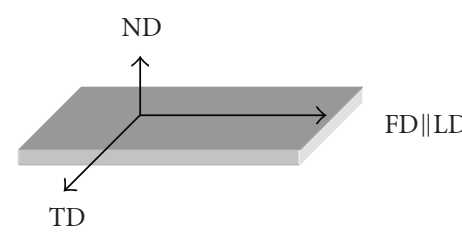

\author{
ND: Sample normal \\ LD: Sample length \\ TD: Sample transverse \\ FD: Magnetic field direction
}

(c)

FIGURE 5: Inverse pole figures of the samples austenitized at $830^{\circ} \mathrm{C}$ for 50 minutes and cooled at a rate of $2^{\circ} \mathrm{C} / \mathrm{min}$ without $(\mathrm{a})$ and with a 12 T magnetic field (b), and the corresponding sample coordinate system. Note: mrd in the figure means "multiples of random distribution" also called trd "times random distribution".

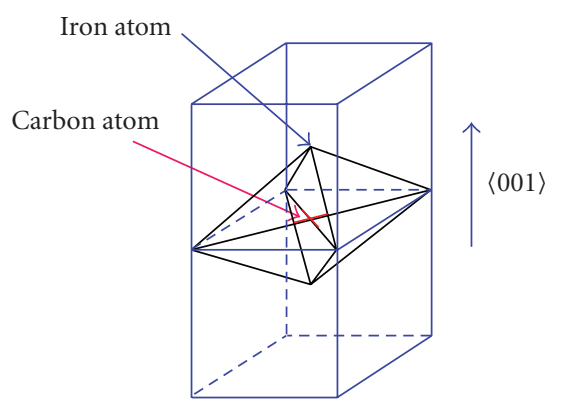

FIGURE 6: Schematic illustration of an octahedral interstice occupied by a carbon atom in bcc Fe [8].

quantitatively for the first time in the present work. The appearance of the proeutectoid ferrite confirms the shift of eutectoid carbon content. Under a $12 \mathrm{~T}$ magnetic field, it shifts from $0.77 \mathrm{wt} . \% \mathrm{C}$ to $0.8287 \mathrm{wt} . \% \mathrm{C}$ (determined from the experimental data). The increase of the pearlite lamellar spacing by the magnetic field qualitatively evidences the increase of the eutectoid temperature. By making use of the quasichemical model to calculate the corresponding activities of Fe and carbon in the related phases and considering the magnetic field influence through magnetic Gibbs free energy determined by the magnetization of the corresponding phases, the new phase equilibrium is calculated. Calculation shows that the increase of the eutectoid temperature by a $12 \mathrm{~T}$ field is $28.97^{\circ} \mathrm{C}$.

In addition, the magnetic field enhances the $\langle 001\rangle$ texture component along the sample normal and transverse directions that are both transverse field directions. This results from the dipolar interaction of the magnetic moments carried by the $\mathrm{Fe}$ atoms. The repelling interaction between $\mathrm{Fe}$ atoms in the transverse direction increases the atomic spacing that mitigates the lattice distortion induced by the solution of carbon atoms in the transverse field direction.

\section{ACKNOWLEDGMENTS}

The authors acknowledge the National Science Fund for Distinguished Young Scholars (no. 50325102), NSF of China (no. 50234020 and 50571024), and PRA MX04-02 Project. They specially thank HASYLAB at DESY, Hamburg, Germany, for synchrotron pole figure measurements.

\section{REFERENCES}

[1] H. Guo and M. Enomoto, "Influence of magnetic fields on alpha/gamma equilibrium in Fe-C(-X) alloys," Material Transactions JIM, vol. 41, no. 8, pp. 911-916, 2000.

[2] J-K. Choi, H. Ohtsuka, Y. Xu, and W-Y. Choo, "Effects of a strong magnetic field on the phase stability of plain carbon steels," Scripta Materialia, vol. 43, no. 3, pp. 221-226, 2000.

[3] H. D. Joo, S. U. Kim, N. S. Shin, and Y. M. Koo, "An effect of high magnetic field on phase transformation in Fe-C system," Materials Letters, vol. 43, no. 5-6, pp. 225-229, 2000.

[4] Y. D. Zhang, C. Esling, M. L. Gong, G. Vincent, X. Zhao, and L. Zuo, "Microstructural features induced by a high magnetic field in a hypereutectoid steel during austenitic decomposition," Scripta Materialia, vol. 54, no. 11, pp. 1897-1900, 2006.

[5] M. Shimotomai and K.-I. Maruta, "Aligned two-phase structures in Fe-C alloys," Scripta Materialia, vol. 42, no. 5, pp. 499503, 2000.

[6] Y. D. Zhang, C. S. He, X. Zhao, L. Zuo, and C. Esling, "Thermodynamic and kinetic characteristics of the austenite-toferrite transformation under high magnetic field in medium carbon steel," Journal of Magnetism and Magnetic Materials, vol. 294, no. 3, pp. 267-272, 2005.

[7] Y. Zhang, C. S. He, X. Zhao, C. Esling, and L. Zuo, "A New approach for rapid annealing of medium carbon steels," Advanced Engineering Materials, vol. 6, no. 5, pp. 310-313, 2004.

[8] Y. D. Zhang, C. Esling, J. S. Lecomte, C. S. He, X. Zhao, and L. Zuo, "Grain boundary characteristics and texture formation in a medium carbon steel during its austenitic decomposition in a high magnetic field," Acta Materialia, vol. 53, no. 19, pp. 5213-5221, 2005. 
[9] L. Wcislak, H. Klein, H. J. Bunge, U. Garbe, T. Tschentscher, and J. R. Schneider, "Texture analysis with high-energy synchrotron radiation," Journal of Applied Crystallography, vol. 35, part 1, pp. 82-95, 2002.

[10] L. Lutterotti, S. Matthies, H.-R. Wenk, A. S. Schultz, and J. W. Richardson, "Combined texture and structure analysis of deformed limestone from time-of-flight neutron diffraction spectra," Journal of Applied Physics, vol. 81, no. 2, pp. 594, 1997.

[11] D. Chateigner, L. Lutterotti, and T. Hansen, "Quantitative phase and texture analysis of ceramic matrix composites," ILL Annual Report 97, Institut Laue-Langevin, Grenoble, France, 1998.

[12] E. du Tremolet de Lacheisserie, D. Gignoux, and M. Schlenker, Magnetism, Springer, New York, NY, USA, 2005.

[13] Y. Mou, T. Y. Hsu, and Z. Xu, "C-C interaction energy in Fe-C alloys," Acta Metallurgica, vol. 34, no. 2, pp. 325-331, 1986.

[14] M. Hillert, Alloy Diffusion and Thermodynamics, Metallurgical Press of China, Bejing, China, 1983.

[15] J. R. Lacher, "The statistics of the hydrogen palladium system," Proceedings of the Cambridge Philosophical Society, vol. 33, pp. 518-523, 1937.

[16] R. H. Fowler and E. A. Guggenheim, Statistical Thermodynamics, Cambridge University Press, New York, NY, USA, 1939.

[17] H. I. Aaronson, H. A. Domian, and G. M. Pound, "Thermodynamics of the austenite proeutectoid ferrite transformation. $\mathrm{i}$, Fe-C alloys," Transactions of the Metallurgical Society of AIME, vol. 236, pp. 753-767, 1966.

[18] G. J. Shiflet, J. R. Bradley, and H. I. Aaronson, "A reexamination of the thermodynamics of the proeutectoid ferrite transformation in Fe-C alloys," Metallurgical and Materials Transactions A, vol. 9, no. 7, pp. 999-1008, 1978.

[19] M. Sakoh and M. Shimizu, "Temperature variations of magnetic susceptibility and electronic specific heat for fcc iron," Journal of the Physical Society of Japan, vol. 37, no. 2, p. 565, 1974.

[20] Y. D. Zhang, H. Faraoun, C. Esling, L. Zuo, and H. Aourag, "Paramagnetic susceptibility of ferrite and cementite obtained from ab initio calculations," Journal of Magnetism and Magnetic Materials, vol. 299, no. 1, pp. 64-69, 2006.

[21] D. Jile, Introduction to Magnetism and Magnetic Materials, Chapman \& Hall, London, UK, 1991.

[22] R. L. Orr and J. Chipman, "Thermodynamic functions of iron," Transactions of the Metallurgical Society of AIME, vol. 239, pp. 630-633, 1967. 